\title{
Interaction of genotype and environment: effect of strain and housing conditions on cognitive behavior in rodent models of schizophrenia
}

\author{
Karly M. Turner ${ }^{1}$ and Thomas H. J. Burne ${ }^{1,2 *}$ \\ ' Queensland Brain Institute, The University of Queensland, St. Lucia, Brisbane, QLD, Australia \\ ${ }^{2}$ Queensland Centre for Mental Health Research, The Park Centre for Mental Health, Wacol, OLD, Australia
}

Edited by:

Tim Karl, Neuroscience Research

Australia, Australia

Reviewed by:

Haim Einat, Tel Aviv-Yaffo Academic

College, Israel

Alicia Izquierdo, University of

California, Los Angeles, USA

*Correspondence:

Thomas H. J. Burne, Queensland Brain Institute, The University of

Queensland, St Lucia, QLD 4072,

Australia

e-mail: t.burne@uq.edu.au

\begin{abstract}
Schizophrenia is associated with many genetic and environmental risk factors and there is growing evidence that the interactions between genetic and environmental "hits" are critical for disease onset. Animal models of schizophrenia have traditionally used specific strain and housing conditions to test potential risk factors. As the field moves towards testing gene $(G) \times$ environment $(E)$ interactions the impact of these choices should be considered. Given the surge of research focused on cognitive deficits, we have examined studies of cognition in rodents from the perspective of GxE interactions, in which strain or housing manipulations have been varied. Behavior is clearly altered by these factors, yet few animal models of schizophrenia have investigated cognitive deficits using different strain and housing conditions. It is important to recognise the large variation in behavior observed when using different strain and housing combinations because GxE interactions may mask or exacerbate cognitive outcomes. Further consideration will improve our understanding of GxE interactions and the underlying neurobiology of cognitive impairments in neuropsychiatric disorders.
\end{abstract}

Keywords: background strain, cognition, enriched environment, schizophrenia, animal model

\section{INTRODUCTION}

Schizophrenia is a complex group of disorders in which genetic vulnerability may lead to greater sensitivity to adverse environmental conditions (Bayer et al., 1999; van Os et al., 2008, 2010; Tost and Meyer-Lindenberg, 2012). Psychiatric epidemiology has provided clues about biologically plausible combinations of genetic and environmental risk factors for the neuroscience field to examine (Caspi and Moffitt, 2006; Meyer and Feldon, 2010). For example, being raised in an urban environment has repeatedly been linked to an increase in psychotic symptoms, however this risk is amplified in individuals with a genetic predisposition to psychosis (van Os et al., 2004; Krabbendam and van Os, 2005; Spauwen et al., 2006; Weiser et al., 2007). Unravelling the neurobiological changes that lead to vulnerable or resilient phenotypes may provide important information about how gene $(G)$ $\mathrm{x}$ environment (E) interactions occur and provide clues for the research community. Rodents have been used to model biologically plausible risk factors and we are beginning to appreciate the complexity of GxE interactions on outcomes relevant to schizophrenia. With the recent focus on measuring cognitive deficits in rodent models (Jentsch, 2003; Kellendonk et al., 2009; Young et al., 2009; Keeler and Robbins, 2011; Bussey et al., 2012) and the known influence of strain and housing conditions on cognitive measures (Chapillon et al., 2002; Harker and Whishaw, 2002; Wolff et al., 2002; Pena et al., 2009; Simpson and Kelly, 2011), it is important to consider whether schizophrenia-related outcomes are dependent on the strain or housing conditions used.
Currently there is a lack of animal models of schizophrenia investigating these GxE interactions on cognitive outcomes. For example, a PubMed search using the terms "strain", "housing", "schizophrenia", "cognition" and "animal model" returned no results; substituting "strain" for "gene", and "housing" for "environment" or "enrichment" only returned seven research articles although none in which housing conditions were compared. Guidelines for cognitive testing in rodents have been established to improve the progression of novel drug treatments, and the use of animal models to examine GxE interactions on established cognitive tests are needed to bridge the translational gap. The next challenge is, therefore, to develop animal models to test the hypothesis that GxE interactions affect cognitive behavior in animal models of schizophrenia. This article focuses on the consequences of strain and housing conditions on cognitive outcomes in rodent models of schizophrenia and how these factors may be useful in modeling GxE interactions.

\section{MODELING THE COGNITIVE DEFICITS IN SCHIZOPHRENIA}

Cognitive deficits are a core symptom group associated with schizophrenia and are the strongest predictor of functional patient outcomes (Green et al., 2000). While cognitive remediation techniques are beneficial, current drug treatments to improve cognitive deficits are largely ineffective and the failure to translate drug findings from animal models to clinical settings has impeded progress (Pratt et al., 2012). To guide future research an initiative of the NIMH was formed, Measurement and Treatment Research 
to Improve Cognition in Schizophrenia (MATRICS), to make suggestions for the development of cognitive testing in animal models of schizophrenia (Green et al., 2004; Young et al., 2009). Based on the core cognitive deficits found in patients with schizophrenia seven cognitive domains were identified including working memory and attention/vigilance (Green et al., 2004). From these domains various clinical tests were selected by the follow-up group, Cognitive Neuroscience Treatment Research to Improve Cognition in Schizophrenia (CNTRICS), to be used in validating drug efficacy and to improve consistency between research groups (Carter and Barch, 2007). In order to bridge the translational gap, tests used in animal models have also been considered and selected for future use and development (Gilmour et al., 2012; Lustig et al., 2012). Domains such as verbal learning and memory cannot be translated to rodents, however processes such as attention, memory and executive control can be measured in a number of ways (Powell and Geyer, 2007) The tests selected for rodents that best reflect the cognitive constructs measured in patients include the five choice serial reaction time task (5CSRTT) (Robbins, 2002) and sustained attention task (dSAT) for measuring attention (Lustig et al., 2012), the attentional setshifting task (ASST) (Birrell and Brown, 2000) and reversal learning (Izquierdo and Jentsch, 2012) as measures of executive control and the radial arm maze (RAM) and delayed match to position (DMTP) task (Dudchenko, 2004), which provide the best assessment of working memory.

The CNTRICS panel reviewed the use of these tests in both rats and mice, however the selection of species and strain should be determined based on suitability for the experimental manipulation and the cognitive test being implemented (Young et al., 2009). The use of non-human primates may also be warranted where processes need to be defined differently for humans and rodents, such as in tests of working memory (Castner et al., 2004). For example, GxE interactions were examined on cognitive outcomes in an animal model of schizophrenia using catecholo-methyl transferase (COMT) knockout mice and the 5C-SRTT (Papaleo et al., 2012). At baseline there was no effect of sex or genotype on cognitive performance, however by manipulating the inter-trial interval, measures of impulsivity were found to differ by sex and genotype. After a mild stressor, males had impaired performance in terms of accuracy and impulsivity measures and this was particularly so for males with reduced $(+/-)$ and absent $(-/-)$ COMT. Other measures were found to differ based on sex and genotype only after reducing motivation. This study illustrates that phenotypes based on sex or genotype may not be readily apparent, however, differences were revealed after manipulating environmental conditions. These findings are in agreement with the suggestion that genes alone do not lead to schizophrenia, but they may predispose an individual to greater vulnerability following exposure to certain environmental insults or "hits".

\section{ENVIRONMENTAL CONDITIONS AND COGNITION}

Epidemiological evidence for the role of environmental factors suggests that housing environment may be an important factor for modeling schizophrenia in rodents (Mcdonald and Murray, 2000). Housing conditions can have a significant influence on rodent behavior and have been used to induce stress or anxiety and to alter cognitive development (van Praag et al., 2000; Nithianantharajah and Hannan, 2006; Burrows et al., 2011; Simpson and Kelly, 2011). Environmental enrichment has been incorporated to enhance sensory and motor experience through the inclusion of novel objects, expanded caging and larger social groups (van Praag et al., 2000). Environmental enrichment has been linked to a number of brain-related outcomes, such as increased brain weight, increased branching and synapse formation in the cortex, increased expression of brain-derived neurotrophic factor (BDNF), glial-cell-derived neurotrophic factor (GDNF) and nerve growth factor (NGF) and increased acetylcholine levels (see review by van Praag et al., 2000). Many of these factors are likely to affect cognitive functioning, for example NGF and BDNF are both known to play a role in learning, while acetylcholine levels have been shown to correlate with attentional performance in rodents (St Peters et al., 2011). Housing conditions have been difficult to standardise across research groups, particularly when enrichment is used. Rather than viewing this noise as a nuisance, it could be seen as an opportunity to investigate how environmental conditions interact with proposed risk factors (Toth et al., 2011).

Rodents reared in more stimulating conditions often acquire tasks after fewer trials (Park et al., 1992), have reduced age-related deficits (Soffie et al., 1999; Harati et al., 2011) and recover from injury faster (Hicks et al., 2002; see Pena et al., 2009). This may indicate phenotypes are being rescued in enriched environments or that deficits only develop in a deprived environment. In some cases, such as animal models of depression, standard housing may be largely contributing to the phenotype, possibly by reducing an animal's compensatory ability to deal with additional challenges (Brenes et al., 2009). The brain may require stimulation beyond that provided in standard housing to develop sufficient connectivity and functionality to detect higher order cognitive deficits. Whether enrichment should be considered as a therapeutic intervention or the standard conditions required for developing a "normal" brain continues to be debated (Wurbel, 2001).

\section{GENETIC BACKGROUND AND COGNITIVE PERFORMANCE}

Mutant mouse models have been used to investigate other key candidate genes linked to schizophrenia (Chen et al., 2006). Despite the availability of tasks for cognitive testing in rodents, a recent review by Arguello and Gogos (2010) did not report any mutant mouse models in which the "top 30" genes linked to schizophrenia had been tested on an attentional paradigm. Considering the need to investigate cognitive symptoms in animal models, there is an obvious gap that needs to be addressed. The genetic risk for schizophrenia is likely to be the result of hundreds or even thousands of genes of small effect (Wray and Visscher, 2010). Systematically testing each individual mutation is unlikely to replicate the disorder, nor is this approach feasible. However, specific genetic mutants may be useful for identifying the origin of cognitive endophenotypes of schizophrenia (see review by Kellendonk et al., 2009). While using single gene mutants provides information about a particular gene of interest (Papaleo et al., 2012), the polygenic nature of schizophrenia may be better modeled by comparing different strains. 
Strain-dependent changes in behavior have been observed on many cognitive tasks and in response to drugs; but these changes are also dependent on the manipulation applied (Andrews et al., 1995; Schmitt and Hiemke, 1998; Mirza and Bright, 2001; Harker and Whishaw, 2002; Wahlsten et al., 2003; Zamudio et al., 2005; Higgins et al., 2007). For example, a widely used task in animal models of schizophrenia, pre-pulse inhibition (PPI) of the acoustic startle response, is a well validated test of sensorimotor gating but results are known to vary depending on the background strain (Rigdon, 1990; Glowa and Hansen, 1994; Varty and Higgins, 1994; Varty et al., 1999; Swerdlow et al., 2001; van den Buuse, 2003). Given the variability on this pre-attentive task, it is not surprising that strain differences have also been reported using more sophisticated cognitive tasks, such as the 5C-SRTT (Didriksen and Christensen, 1993; Mirza and Bright, 2001; Higgins et al., 2007; Auclair et al., 2009). These studies also demonstrate the variability between studies using the same strain, which may be due to variation in the protocol used or the source of the strain (Andrews, 1996; Karl et al., 2011).

Rat models of schizophrenia have been developed predominantly using two albino strains, however the reasons for these selections are not always obvious. Furthermore, studies of schizophrenia-related manipulations comparing rat strains are lacking. The neonatal ventral hippocampal lesion model was compared in the outbred Sprague-Dawley (SD) and two inbred strains, Lewis and Fischer 344, which differed in stress responsivity (Lipska and Weinberger, 1995). For example, SD and Lewis rats show habituation of the hypothalamic-pituitary-adrenal axis (HPA) response to a repeated restraint stress paradigm, whereas F344 rats do not habituate within or between stress-inducing sessions (Dhabhar et al., 1997). As predicted the hyper-responsive F344 strain showed greater behavioral vulnerability to the neonatal lesion, while the hypo-responsive Lewis rats showed greater resistance when both were compared to the SD strain. Thus, stress responsivity is a critical consideration both for models utilising stressful manipulations and for the interpretation of behavioral results from different strains (Faraday, 2002). Spontaneous and amphetamine-induced hyper locomotion varied across development with strain, indicating genetic predisposition has a critical role in determining the phenotype derived from this neurodevelopmental model, although cognitive outcomes were not assessed in this study (Lipska and Weinberger, 1995).

\section{GENE X ENVIRONMENT INTERACTIONS AND COGNITIVE ENDOPHENOTYPES}

The focus of GxE interaction studies in animal models of schizophrenia has taken advantage of the genetic tools available in mice, comparing mutant and control animals after adverse environmental exposures such as immune activation, stress or drug administration (Kannan et al., 2013). The influence of enriched housing conditions on rodent models of schizophrenia has been addressed by only a few studies (Karl et al., 2007; McOmish et al., 2008; Ishihama et al., 2010). However, the neurological and behavioral effects of environmental enrichment have been assessed in a range of other animal models including Huntington's disease, Alzheimer's disease, Parkinson's disease, Epilepsy and drug addiction (Bezard et al., 2003; see review by Nithianantharajah and Hannan, 2006; Laviola et al., 2008). For example, the influence of environmental enrichment has been shown using the transgenic mouse model of Huntington's disease (van Dellen et al., 2000). This neurodegenerative condition has a genetic cause, yet mice housed in enriched cages show delayed onset and progression of both the motor and cognitive deficits compared to standard housed controls (Hockly et al., 2002; Nithianantharajah and Hannan, 2006; Pang et al., 2006). Using animal models of schizophrenia, it will not only be important to address the detrimental effects of the environment, but also conditions that have a protective influence (Takuma et al., 2011; Pang and Hannan, 2013).

With the aim of developing biologically-relevant animal models of schizophrenia, studies using a GxE approach are rapidly emerging (Millstein et al., 2006; Millstein and Holmes, 2007; Oliver and Davies, 2009; Desbonnet et al., 2012; Hida et al., 2013; Petrovszki et al., 2013). Prenatal stress followed by acute stress during adulthood was used in three rat strains to examine how genetic background interacted with adverse environmental conditions to alter hippocampal gene expression (Neeley et al., 2011b). Five relevant genes (Nr3c1, Chrna7, Grin2b, Bdnf, Tnf $\alpha$ ) were found to be altered by either strain or stress treatments, however changes were inconsistent across strains indicating a modulatory role of genotype. A second experiment comparing these strains using a stress protocol found that changes in $B d n f$ expression and associated pathways were also strain dependent (Neeley et al., 2011a). These studies demonstrate the importance of strain selection and genetic diversity in understanding GxE interactions.

In another recent study, rats were exposed to two commonly used risk factors, post weaning social isolation and chronic ketamine treatment, and selectively bred based on behavioral deficits relevant to schizophrenia to produce a vulnerable sub-strain (Petrovszki et al., 2013). After 15 generations, four groups were compared on three behavioral tests and the results were accumulated into an overall score. Rats with a standard genetic background raised under standard conditions were used as a control group. The environment-only group consisted of genetically-naïve rats that were then isolated and treated with ketamine. Rats from the selectively bred vulnerable sub-strain that were raised under standard conditions were used as the geneticonly group. And finally rats from the vulnerable sub-strain that also underwent social isolation and chronic ketamine treatment were used to investigate the GxE interaction. The GxE group scored the highest on schizophrenia-relevant deficits and the control group scored the lowest, indicating that both genetic and environmental insults were important. The behavioral tests used assess nociception, sensorimotor gating and recognition memory, which do not address the key cognitive domains identified by CNTRICS and therefore further work would be required to understand the influence of these manipulations on cognitive deficits relevant to schizophrenia. Nevertheless, this study does present a new way of investigating previously tested risk factors.

\section{FUTURE RECOMMENDATIONS}

A recent review of mouse models of $\mathrm{GxE}$ interactions relevant to schizophrenia has discussed a comprehensive list of weaknesses to be addressed by future studies (Kannan et al., 2013). The 
authors suggested standardising strain and housing conditions to reduce variability between studies. However, genetic and environmental choices clearly alter outcomes relevant to schizophrenia and phenotypes may only be detected under specific strain or housing conditions. Furthermore, the way genetic and environmental conditions interact to protect or exacerbate phenotypes is of key importance in understanding the pathways that lead to schizophrenia.

Investigating genetic changes, such as mutant mouse models, may be easily replicated across laboratories, however environmental manipulations are more difficult to standardise. For example, wild type mice show different behavioral phenotypes when tested under similar conditions but at different laboratories (Crabbe et al., 1999). More recently, heterozygous neuregulin mutant mice showed different behavioral phenotypes when tested in different laboratories, despite being on the same genetic background (Karl et al., 2011). Although these differences may be unavoidable, it is recommended the housing conditions of rodents be clearly stated in research methods. Unfortunately many articles do not list the forms of enrichment used (such as type of bedding, shelters, wood chews and tubes) however these should be indicated even if considered to represent "standard" housing conditions. Recommending a standardised enrichment protocol would reduce variability between experiments, but would also limit the scope of enrichment studies (Wurbel, 2002). Protocol design should take into consideration the species-specific relevance of environmental changes, the timing and duration of exposure, the ethical implications and the reproducibility of the chosen design. Therefore, optimal enrichment conditions should be selected based on experimental aims.

Future studies could take a number of directions, including the use of GxG and ExE studies to identify the influence of genetic and environmental factors; as well as understanding the mechanisms that lead to increased vulnerability (Giovanoli et al., 2013). To more fully assess the effects of GxE interactions on cognitive endophenotypes, the field also needs to improve the range of

\section{REFERENCES}

Andrews, J. S. (1996). Possible confounding influence of strain, age and gender on cognitive performance in rats. Brain Res. Cogn. Brain Res. 3, 251-267. doi: 10.1016/09266410(96)00011-0

Andrews, J. S., Jansen, J. H., Linders, S., Princen, A., and Broekkamp, C. L. (1995). Performance of four different rat strains in the autoshaping, two-object discrimination, and swim maze tests of learning and memory. Physiol. Behav. 57, 785-790. doi: 10.1016/00319384(94)00336-X

Arguello, P. A., and Gogos, J. A. (2010). Cognition in mouse models of schizophrenia susceptibility genes. Schizophr. Bull. 36, 289-300. doi: 10.1093/schbul/sbp153

Auclair, A. L., Besnard, J., NewmanTancredi, A., and Depoortere, R.
(2009). The five choice serial reaction time task: comparison between Sprague-Dawley and Long-Evans rats on acquisition of task, and sensitivity to phencyclidine. Pharmacol. Biochem. Behav. 92, 363-369. doi: 10.1016/j.pbb.2009.01.005

Bayer, T. A., Falkai, P., and Maier, W. (1999). Genetic and non-genetic vulnerability factors in schizophrenia: the basis of the "two hit hypothesis”. J. Psychiatr. Res. 33, 543548. doi: 10.1016/s0022-3956(99) 00039-4

Bezard, E., Dovero, S., Belin, D., Duconger, S., Jackson-Lewis, V., Przedborski, S., et al. (2003). Enriched environment confers resistance to 1-methyl-4-phenyl1,2,3,6-tetrahydropyridine and cocaine: involvement of dopamine transporter and trophic factors. $J$. Neurosci. 23, 10999-11007. the behavioral tasks available. The potential therapeutic benefit of improved animal models may be limited by the sensitivity of the behavioral measures employed. Incorporating GxE clues from epidemiology into our animal models, and improving assessment techniques will advance our understanding of schizophrenia.

\section{CONCLUSION}

There is clear evidence to show that genetic and environmental conditions alter cognitive outcomes in rodents. However, the lack of studies comparing cognitive deficits in rodent models of schizophrenia using different strain and housing conditions is surprising. Schizophrenia develops from the complex interaction of GxE and we need to incorporate this complexity into animal models to understand the etiology of schizophrenia. Although it is difficult to recapitulate complex disorders, such as schizophrenia, in a rodent model, the use of endophenotypes in carefully controlled experiments may allow us to better understand some of the mechanisms behind GxE interactions. Current animal models are falling short of replicating the complex suite of risk factors implicated in schizophrenia and using different strains or housing conditions may provide an accessible stepping stone towards understanding altered brain development. Given the infancy of GxE interaction research in animal models of schizophrenia, manipulating these factors in existing and novel animal models will be informative in terms of GxE interactions. GxE interaction models will be particularly informative for understanding the role of vulnerable and resilient phenotypes in determining the influence of secondary "hits" on cognitive outcomes in schizophrenia.

\section{ACKNOWLEDGMENTS}

Thomas H. J. Burne acknowledges support from Queensland Health and the National Health and Medical Research Council of Australia (Project Grant no. 511066). Karly M. Turner was supported by an Australian Postgraduate Award and a Queensland Government Smart Futures PhD Scholarship.
Birrell, J. M., and Brown, V. J. (2000). Medial frontal cortex mediates perceptual attentional set shifting in the rat. J. Neurosci. 20, 4320-4324.

Brenes, J. C., Padilla, M., and Fornaguera, J. (2009). A detailed analysis of open-field habituation and behavioral and neurochemical antidepressant-like effects in postweaning enriched rats. Behav. Brain Res. 197, 125-137. doi: 10.1016/j.bbr.2008.08.014

Burrows, E. L., Mcomish, C. E., and Hannan, A. J. (2011). Geneenvironment interactions and construct validity in preclinical models of psychiatric disorders. Prog. Neuropsychopharmacol. Biol. Psychiatry 35, 1376-1382. doi: 10.1016/j.pnpbp.2010.12.011

Bussey, T. J., Holmes, A., Lyon, L., Mar, A. C., Mcallister, K. A. L., Nithianantharajah, J., et al. (2012).
New translational assays for preclinical modelling of cognition in schizophrenia: the touchscreen testing method for mice and rats. Neuropharmacology 62, 1191-1203. doi: 10.1016/j.neuropharm.2011. 04.011

Carter, C. S., and Barch, D. M. (2007). Cognitive neurosciencebased approaches to measuring and improving treatment effects on cognition in schizophrenia: the CNTRICS initiative. Schizophr. Bull. 33, 1131-1137. doi: 10.1093/schbul/sbm081

Caspi, A., and Moffitt, T. E. (2006). Gene-environment interactions in psychiatry: joining forces with neuroscience. Nat. Rev. Neurosci. 7, 583-590. doi: 10.1038/nrn1925

Castner, S. A., Goldman-Rakic, P. S., and Williams, G. V. (2004). Animal models of working memory: 
insights for targeting cognitive dysfunction in schizophrenia. Psychopharmacology 174, 111-125. doi: 10.1007/s00213-003-1710-9

Chapillon, P., Patin, V., Roy, V., Vincent, A., and Caston, J. (2002). Effects of pre- and postnatal stimulation on developmental, emotional, and cognitive aspects in rodents: a review. Dev. Psychobiol. 41, 373-387. doi: 10.1002/dev.10066

Chen, J., Lipska, B. K., and Weinberger, D. R. (2006). Genetic mouse models of schizophrenia: from hypothesis-based to susceptibility gene-based models. Biol. Psychiatry 59, 1180-1188. doi: 10.1016/j.biopsych.2006.02.024

Crabbe, J. C., Wahlsten, D., and Dudek, B. C. (1999). Genetics of mouse behavior: interactions with laboratory environment. Science 284, 1670-1672. doi: 10.1126/science.284.5420.1670

Desbonnet, L., O’tuathaigh, C., Clarke, G., O'leary, C., Petit, E., Clarke, N., et al. (2012). Phenotypic effects of repeated psychosocial stress during adolescence in mice mutant for the schizophrenia risk gene neuregulin-1: a putative model of gene $\mathrm{x}$ environment interaction. Brain Behav. Immun. 26, 660671. doi: 10.1016/s0920-9964(12) 70331-6

Dhabhar, F. S., Mcewen, B. S., and Spencer, R. L. (1997). Adaptation to prolonged or repeated stresscomparison between rat strains showing intrinsic differences in reactivity to acute stress. $\mathrm{Neu}$ roendocrinology 65, 360-368. doi: 10.1159/000127196

Didriksen, M., and Christensen, A. V. (1993). Differences in performance in three strains of rats in a 5-choice serial reaction time task. Pharmacol. Toxicol. 72, 66-68. doi: 10.1111/j.16000773.1993.tb01341.x

Dudchenko, P. A. (2004). An overview of the tasks used to test working memory in rodents. Neurosci. Biobehav. Rev. 28, 699-709. doi: 10.1016/j.neubiorev.2004.09.002

Faraday, M. M. (2002). Rat sex and strain differences in responses to stress. Physiol. Behav. 75, 507522. doi: 10.1016/s0031-9384(02) 00645-5

Gilmour, G., Arguello, A., Bari, A., Brown, V. J., Carter, C., Floresco, S. B., et al. (2012). Measuring the construct of executive control in schizophrenia: defining and validating translational animal paradigms for discovery research. Neurosci. Biobehav. Rev. doi: 10.1016/j.neubiorev.2012.04.006. [Epub ahead of print]

Giovanoli, S., Engler, H., Engler, A., Richetto, J., Voget, M., Willi, R., et al. (2013). Stress in puberty unmasks latent neuropathological consequences of prenatal immune activation in mice. Science 339, 1095-1099. doi: 10.1126/science. 1228261

Glowa, J. R., and Hansen, C. T. (1994). Differences in response to an acoustic startle stimulus among forty-six rat strains. Behav. Genet. 24, 79-84. doi: 10.1007/bf01067931

Green, M. F., Kern, R. S., Braff, D. L., and Mintz, J. (2000). Neurocognitive deficits and functional outcome in schizophrenia: are we measuring the "right stuff"? Schizophr. Bull. 26, 119-136. doi: 10.1093/oxfordjournals.schbul.a033430

Green, M. F., Nuechterlein, K. H., Gold, J. M., Barch, D. M. Cohen, J., Essock, S., et al. (2004). Approaching a consensus cognitive battery for clinical trials in schizophrenia: the NIMHMATRICS conference to select cognitive domains and test criteria. Biol. Psychiatry 56, 301-307. doi: 10.1016/j.biopsych.2004.06.023

Harati, H., Majchrzak, M., Cosquer, B., Galani, R., Kelche, C., Cassel, J. C., et al. (2011). Attention and memory in aged rats: impact of lifelong environmental enrichment. Neurobiol. Aging 32, 718-736. doi: 10.1016/j.neurobiolaging.2009. 03.012

Harker, K. T., and Whishaw, I. Q. (2002). Place and matching-toplace spatial learning affected by rat inbreeding (Dark-Agouti, Fischer 344) and albinism (Wistar, Sprague-Dawley) but not domestication (wild rat vs. Long-Evans, Fischer-Norway). Behav. Brain Res. 134, 467-477. doi: 10.1016/s01664328(02)00083-9

Hicks, R. R., Zhang, L., Atkinson, A., Stevenon, M., Veneracion, M., and Seroogy, K. B. (2002). Environmental enrichment attenuates cognitive deficits, but does not alter neurotrophin gene expression in the hippocampus following lateral fluid percussion brain injury. Neuroscience 112, 631637. doi: 10.1016/s0306-4522(02) 00104-5

Hida, H., Mouri, A., and Noda, Y. (2013). Behavioral phenotypes in schizophrenic animal models with multiple combinations of genetic and environmental factors. J. Pharmacol. Sci. 121, 185-191. doi: 10.1254/jphs.12r15cp
Higgins, G. A., Grzelak, M. E., Pond, A. J., Cohen-Williams, M. E., Hodgson, R. A., and Varty, G. B. (2007). The effect of caffeine to increase reaction time in the rat during a test of attention is mediated through antagonism of adenosine A2A receptors. Behav. Brain Res. 185, 32 42. doi: 10.1016/j.bbr.2007.07.013

Hockly, E., Cordery, P. M., Woodman, B., Mahal, A., van Dellen, A., Blakemore, C., et al. (2002). Environmental enrichment slows disease progression in R6/2 Huntington's disease mice. Ann. Neurol. 51, 235-242. doi: 10.1097/01253086-20022604000009

Ishihama, T., Ago, Y., Shintani, N., Hashimoto, H., Baba, A., Takuma, K., et al. (2010). Environmental factors during early developmental period influence psychobehavioral abnormalities in adult PACAP-deficient mice. Behav. Brain Res. 209, 274-280. doi: 10.1016/j.bbr.2010.02.009

Izquierdo, A., and Jentsch, J. D. (2012). Reversal learning as a measure of impulsive and compulsive behavior in addictions. Psychopharmacology (Berl) 219, 607-620. doi: 10.1007/s00213-011-2579-7

Jentsch, J. D. (2003). Pre-clinical models of cognitive dysfunction in schizophrenia: new avenues to addressing unmet needs. Clin. Neurosci. Res. 3, 303-313. doi: 10.1016/ j.cnr.2003.10.011

Kannan, G., Sawa, A., and Pletnikov, M. V. (2013). Mouse models of gene-environment interactions in schizophrenia. Neurobiol. Dis. 57, 5-11. doi: 10.1016/j.nbd.2013. 05.012

Karl, T., Burne, T. H., van den Buuse, M., and Chesworth, R. (2011) Do transmembrane domain neuregulin 1 mutant mice exhibit a reliable sensorimotor gating deficit? Behav. Brain Res. 223, 336-341. doi: 10.1016/j.bbr.2011.04.051

Karl, T., Duffy, L., Scimone, A., Harvey, R. P., and Schofield, P. R. (2007). Altered motor activity, exploration and anxiety in heterozygous neuregulin 1 mutant mice: implications for understanding schizophrenia. Genes Brain Behav. 6, 677-687. doi: 10.1111/j.1601-183x.2006.00298.x

Keeler, J. F., and Robbins, T. W. (2011). Translating cognition from animals to humans. Biochem. Pharmacol. 81, 1356-1366. doi: 10.1016/j.bcp.2010.12.028

Kellendonk, C., Simpson, E. H., and Kandel, E. R. (2009). Modeling cognitive endophenotypes of schizophrenia in mice. Trends
Neurosci. 32, 347-358. doi: 10.1016/ j.tins.2009.02.003

Krabbendam, L., and van Os, J. (2005). Schizophrenia and urbanicity: a major environmental influence-conditional on genetic risk. Schizophr. Bull. 31, 795-799. doi: 10.1093/schbul/sbi060

Laviola, G., Hannan, A. J., Macri, S., Solinas, M., and Jaber, M. (2008) Effects of enriched environment on animal models of neurodegenerative diseases and psychiatric disorders. Neurobiol. Dis. 31, 159-168. doi: 10.1016/j.nbd.2008.05.001

Lipska, B. K., and Weinberger, D. R. (1995). Genetic variation in vulnerability to the behavioral effects of neonatal hippocampal damage in rats. Proc. Natl. Acad. Sci. U S A 92, 8906-8910. doi: 10.1073/pnas.92.19.8906

Lustig, C., Kozak, R., Sarter, M., Young, J. W., and Robbins, T. W. (2012). CNTRICS final animal model task selection: control of attention. Neurosci. Biobehav. Rev. doi: 10.1016/j.neubiorev.2012.05.009. [Epub ahead of print]

Mcdonald, C., and Murray, R. M. (2000). Early and late environmental risk factors for schizophrenia. Brain Res. Brain Res. Rev. 31, 130-137. doi: 10.1016/s01650173(99)00030-2

McOmish, C. E., Burrows, E., Howard, M., Scarr, E., Kim, D., Shin, H. S., et al. (2008). Phospholipase C-betal knockout mice exhibit endophenotypes modeling schizophrenia which are rescued by environmental enrichment and clozapine administration. Mol. Psychiatry 13, 661-672. doi: 10.1038/sj.mp.4002046

Meyer, U., and Feldon, J. (2010) Epidemiology-driven neurodevelopmental animal models of schizophrenia. Prog. Neurobiol. 90, 285-326. doi: 10.1016/ j.pneurobio.2009.10.018

Millstein, R. A., and Holmes, A (2007). Effects of repeated maternal separation on anxiety- and depression-related phenotypes in different mouse strains. Neurosci. Biobehav. Rev. 31, 3-17. doi: 10.1016/j.neubiorev.2006.05.003

Millstein, R. A., Ralph, R. J., Yang, R. J., and Holmes, A. (2006) Effects of repeated maternal separation on prepulse inhibition of startle across inbred mouse strains. Genes Brain Behav. 5, 346-354. doi: 10.1111/j.1601-183x.2005.00172.x

Mirza, N. R., and Bright, J. L. (2001). Nicotine-induced enhancements in the five-choice serial reaction time task in rats are strain-dependent. 
Psychopharmacology (Berl) 154, 812. doi: $10.1007 / \mathrm{s} 002130000605$

Neeley, E. W., Berger, R., Koenig, J. I., and Leonard, S. (2011a). Prenatal stress differentially alters brainderived neurotrophic factor expression and signaling across rat strains. Neuroscience 187, 24-35. doi: 10.1016/j.neuroscience.2011.03.065

Neeley, E. W., Berger, R., Koenig, J. I., and Leonard, S. (2011b). Strain dependent effects of prenatal stress on gene expression in the rat hippocampus. Physiol. Behav. 104, 334-339. doi: 10.1016/j.physbeh.2011.02.032

Nithianantharajah, J., and Hannan, A. J. (2006). Enriched environments, experience-dependent plasticity and disorders of the nervous system. Nat. Rev. Neurosci. 7, 697-709. doi: 10.1038/nrn1970

Oliver, P. L., and Davies, K. E. (2009). Interaction between environmental and genetic factors modulates schizophrenic endophenotypes in the Snap-25 mouse mutant blinddrunk. Hum. Mol. Genet. 18, 45764589. doi: $10.1093 / \mathrm{hmg} / \mathrm{ddp} 425$

Pang, T. Y., and Hannan, A. J. (2013). Enhancement of cognitive function in models of brain disease through environmental enrichment and physical activity. Neuropharmacology 64, 515-528. doi: 10.1016/j.neuropharm.2012.06.029

Pang, T. Y., Stam, N. C., Nithianantharajah, J., Howard, M. L., and Hannan, A. J. (2006). Differential effects of voluntary physical exercise on behavioral and brain-derived neurotrophic factor expression deficits in Huntington's disease transgenic mice. Neuroscience 141，569-584. doi: 10.1016/j.neuroscience.2006.04.013

Papaleo, F., Erickson, L., Liu, G., Chen, J., and Weinberger, D. R. (2012). Effects of sex and COMT genotype on environmentally modulated cognitive control in mice. Proc. Natl. Acad. Sci. U S A 109, 20160-20165. doi: 10.1073/pnas.1214397109

Park, G. A., Pappas, B. A., Murtha, S. M., and Ally, A. (1992). Enriched environment primes forebrain choline acetyltransferase activity to respond to learning experience. Neurosci. Lett. 143, 259-262. doi: 10.1016/0304-3940(92)90278-f

Pena, Y., Prunell, M., Rotllant, D., Armario, A., and Escorihuela, R. M. (2009). Enduring effects of environmental enrichment from weaning to adulthood on pituitary-adrenal function, prepulse inhibition and learning in male and female rats. Psychoneu- roendocrinology 34, 1390-1404. doi: 10.1016/j.psyneuen.2009.04.019

Petrovszki, Z., Adam, G., Tuboly, G., Kekesi, G., Benedek, G., Keri, S. et al. (2013). Characterization of gene-environment interactions by behavioral profiling of selectively bred rats: the effect of NMDA receptor inhibition and social isolation. Behav. Brain Res. 240, 134-145. doi: 10.1016/j.bbr.2012.11.022

Powell, S. B., and Geyer, M. A. (2007). Overview of animal models of schizophrenia. Curr. Protoc. Neurosci. 39 (Suppl.), 9.24.2129.24.20. doi: 10.1002/0471142301. ns0924s39

Pratt, J., Winchester, C., Dawson, N., and Morris, B. (2012). Advancing schizophrenia drug discovery: optimizing rodent models to bridge the translational gap. Nat. Rev. Drug Discov. 11, 560-579. doi: 10.1038/nrd3649

Rigdon, G. C. (1990). Differential effects of apomorphine on prepulse inhibition of acoustic startle reflex in two rat strains. Psychopharmacology (Berl) 102, 419-421. doi: 10.1007/bf02244115

Robbins, T. W. (2002). The 5-choice serial reaction time task: behavioral pharmacology and functional neurochemistry. Psychopharmacology 163, 362-380. doi: 10.1007/s00213002-1154-7

Schmitt, U., and Hiemke, C. (1998). Strain differences in open-field and elevated plus-maze behavior of rats without and with pretest handling. Pharmacol. Biochem. Behav. 59, 807-811. doi: 10.1016/s00913057(97)00502-9

Simpson, J., and Kelly, J. P. (2011). The impact of environmental enrichment in laboratory rats-behavioral and neurochemical aspects. Behav. Brain Res. 222, 246-264. doi: 10.1016/j.bbr.2011.04.002

Soffie, M., Hahn, K., Terao, E., and Eclancher, F. (1999). Behavioral and glial changes in old rats following environmental enrichment. Behav. Brain Res. 101, 37-49. doi: 10.1016/s0166-4328(98)00139-9

Spauwen, J., Krabbendam, L., Lieb, R., Wittchen, H. U., and van Os, J. (2006). Evidence that the outcome of developmental expression of psychosis is worse for adolescents growing up in an urban environment. Psychol. Med. 36, 407-415. doi: $10.1017 / \mathrm{s} 0033291705006902$

St Peters, M., Demeter, E., Lustig, C., Bruno, J. P., and Sarter, M. (2011). Enhanced control of attention by stimulating mesolimbiccorticopetal cholinergic circuitry.
J. Neurosci. 31, 9760-9771. doi: 10.1523/jneurosci.1902-11.2011

Swerdlow, N. R., Platten, A., Kim, Y. K., Gaudet, I., Shoemaker, J., Pitcher, L., et al. (2001). Sensitivity to the dopaminergic regulation of prepulse inhibition in rats: evidence for genetic, but not environmental determinants. Pharmacol. Biochem. Behav. 70, 219 226. doi: 10.1016/s0091-3057(01) 00598-6

Takuma, K., Ago, Y., and Matsuda, T. (2011). Preventive effects of an enriched environment on rodent psychiatric disorder models. J. Pharmacol. Sci. 117, 71-76. doi: 10.1254/jphs.11r07cp

Tost, H., and Meyer-Lindenberg, A. (2012). Puzzling over schizophrenia: schizophrenia, social environment and the brain. Nat. Med. 18, 211-213. doi: 10.1038/ nm.2671

Toth, L. A., Kregel, K., Leon, L., and Musch, T. I. (2011). Environmental enrichment of laboratory rodents: the answer depends on the question. Comp. Med. 61, 314-321.

van Dellen, A., Blakemore, C., Deacon, R., York, D., and Hannan, A. J. (2000). Delaying the onset of Huntington's in mice. Nature 404, 721722. doi: $10.1038 / 35008142$

van den Buuse, M. (2003). Deficient prepulse inhibition of acoustic startle in Hooded-Wistar rats compared with Sprague-Dawley rats. Clin. Exp. Pharmacol. Physiol. 30, 254-261. doi: 10.1046/j.14401681.2003.03823. $\mathrm{x}$

van Os, J., Kenis, G., and Rutten, B. P. (2010). The environment and schizophrenia. Nature 468, 203-212. doi: 10.1038/nature09563

van Os, J., Pedersen, C. B., and Mortensen, P. B. (2004). Confirmation of synergy between urbanicity and familial liability in the causation of psychosis. Am. J. Psychiatry 161, 2312-2314. doi 10.1176/appi.aip.161.12.2312

van Os, J., Rutten, B. P. F., and Poulton, R. (2008). Gene-environment interactions in schizophrenia: review of epidemiological findings and future directions. Schizophr. Bull. 34, 1066-1082. doi: 10.1093/schbul/ $\operatorname{sbn} 117$

van Praag, H., Kempermann, G., and Gage, F. H. (2000). Neural consequences of environmental enrichment. Nat. Rev. Neurosci. 1, 191198. doi: $10.1038 / 35044558$

Varty, G. B., and Higgins, G. A. (1994) Differences between 3 rat strains in sensitivity to prepulse inhibition of an acoustic startle response - influence of apomorphine and phencyclidine pretreatment. $J$. Psychopharmacol. 8, 148-156. doi: 10.1177/026988119400800302

Varty, G. B., Bakshi, V. P., and Geyer, M. A. (1999). M100907, a serotonin 5-HT2A receptor antagonist and putative antipsychotic, blocks dizocilpine-induced prepulse inhibition deficits in SpragueDawley and Wistar rats. Neuropsychopharmacology 20, 311-321. doi: 10.1016/s0893-133x(98)00072-4

Wahlsten, D., Rustay, N. R., Metten, P., and Crabbe, J. C. (2003). In search of a better mouse test. Trends Neurosci. 26, 132-136. doi 10.1016/s0166-2236(03)00033-x

Weiser, M., van Os, J., Reichenberg, A., Rabinowitz, J., Nahon, D. Kravitz, E., et al. (2007). Social and cognitive functioning, urbanicity and risk for schizophrenia. $B r$ J. Psychiatry 191, 320-324. doi 10.1192/bjp.bp.106.031328

Wolff, M., Savova, M., Malleret, G. Segu, L., and Buhot, M. C. (2002). Differential learning abilities of $129 \mathrm{~T} 2 / \mathrm{Sv}$ and $\mathrm{C} 57 \mathrm{BL} / 6 \mathrm{~J}$ mice as assessed in three water maze protocols. Behav. Brain Res. 136, 463-474. doi: $\quad 10.1016 / \mathrm{s} 0166-4328(02)$ 00192-4

Wray, N. R., and Visscher, P. M. (2010). Narrowing the boundaries of the genetic architecture of schizophrenia. Schizophr. Bull. 36, 14-23. doi: $10.1093 / \mathrm{schbul} / \mathrm{sbp} 137$

Wurbel, H. (2001). Ideal homes? Housing effects on rodent brain and behavior. Trends Neurosci. 24, 207-211. doi: 10.1016/s01662236(00)01718-5

Wurbel, H. (2002). Behavioral phenotyping enhanced-beyond (environmental) standardization. Genes Brain Behav. 1, 3-8. doi 10.1046/j.1601-1848.2001.00006.x

Young, J. W., Powell, S. B., Risbrough, V., Marston, H. M., and Geyer, M. A. (2009). Using the MATRICS to guide development of a preclinical cognitive test battery for research in schizophrenia. Pharmacol. Ther. 122, 150-202. doi: 10.1016/j.pharmthera.2009. 02.004

Zamudio, S., Fregoso, T., Miranda, A., De La Cruz, F., and Flores, G. (2005). Strain differences of dopamine receptor levels and dopamine related behaviors in rats. Brain Res. Bull. 65, 339-347. doi: 10.1016/j.brainresbull.2005 01.009

Conflict of Interest Statement: The authors declare that the research was 
conducted in the absence of any commercial or financial relationships that could be construed as a potential conflict of interest.

Received: 01 May 2013; paper pending published: 26 May 2013; accepted:
16 July 2013; published online: 31 July 2013

Citation: Turner KM and Burne TH (2013) Interaction of genotype and environment: effect of strain and housing conditions on cognitive behaviour in rodent models of schizophrenia. Front. Behav. Neurosci. 7:97. doi: 10.3389/fnbeh.2013.00097

Copyright (c) 2013 Turner and Burne. This is an open-access article distributed under the terms of the Creative Commons Attribution License (CC BY). The use, distribution or reproduction in other forums is permitted, provided the original author(s) or licensor are credited and that the original publication in this journal is cited, in accordance with accepted academic practice. No use, distribution or reproduction is permitted which does not comply with these terms. 